
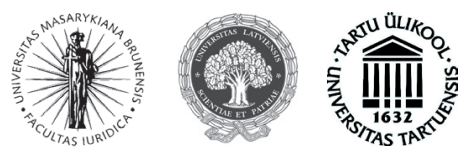

ISSN 1392-6195 (print) ISSN 2029-2058 (online) JURISPRUDENCIJA JURISPRUDENCE 2013, 20(3), p. 951-970.

\title{
NEUE MASSSTÄBE IM EU-ERBRAUCHERSCHUTZRECHT? DIE NEUE VERBRAUCHERRECHTERICHTLINIE 2011/83/EU
}

\author{
Arndt Künnecke \\ Okan Üniversity Istanbul \\ Tuzla Campus, 34959 Tuzla-Istanbul, Turkey \\ Tel.: 00905378334566 \\ E-Mail: kuennecke@web.de
}

Received on 24 of July 2013; accepted on 20 of September 2013

doi:10.13165/JUR-13-20-3-06

Annotation. Nach jahrelangen Beratungen trat die Verbraucherrechterichtlinie 2011/83/EU, die neue Maßstäbe im europäischen Verbraucherschutz setzen sollte, in Kraft und muss von den Mitgliedsstaaten bis zum 13. Dezember 2013 in innerstaatliches Recht umgesetzt und mit den geänderten Vorschriften am 13. Juni 2014 in Kraft gesetzt werden. Dieser Aufsatz erklärt zunächst den Hintergrund der Richtlinie und erörtert deren Abkehr von der bisher vorherrschenden Praxis einer Mindestharmonisierung im Verbraucherschutz hin zum neuen Ansatz einer zielgerichteten Vollharmonisierung. Daran anschließend werden Anwendungsbereich, Begriffe und Inhalt der Richtlinie analysiert, die vor allem in den Bereichen Informationspflichten und Widerrufsrecht bemerkenswerte Reformen mit sich bringt. Unter Berücksichtigung der mit der neuen Verbraucherrechterichtlinie verbundenen Verbesserungen, aber auch ihrer Unzulänglichkeiten, kommt der Autor zum Ergebnis, dass es einer weiteren Harmonisierung des europäischen Verbraucherrechts bedarf und am Ende des Harmonisierungsprozesses ein einheitliches und allgemein verbindliches europäisches Verbraucherschutzrecht stehen sollte.

Keywords: Consumer Rights Protection, Consumer Contract Law, EU-Directives, EU Consumer Law, Full Harmonisation Approach. 


\section{Einleitung}

Die neue EU-Verbraucherrechterichtlinie 2011/83/EU ${ }^{1}$ (hiernach: VRR), die am 22. November 2011 veröffentlicht worden und am 12. Dezember 2011 in Kraft getreten ist, muss von den EU-Mitgliedsstaaten bis zum 13. Dezember 2013 umgesetzt und mit den geänderten Vorschriften am 13. Juni 2014 in Kraft gesetzt werden. ${ }^{2}$ Ihr Ziel ist es, den Verbraucherschutz EU-weit zu verbessern, indem sie das derzeit noch unterschiedliche innerstaatliche Recht für Fernabsatzverträge und außerhalb von Geschäftsräumen geschlossene Verträge sowie andere Arten von Verbraucherverträgen in einem einzigen Dokument zusammenfasst. Entsprechend dem Ziel der Richtlinie soll ein echter Binnenmarkt für Geschäfte zwischen Unternehmen und Verbrauchern durch ein ausgewogenes Verhältnis zwischen einem hohen Verbraucherschutzniveau und der Wettbewerbsfähigkeit erreicht werden. ${ }^{3}$

Am 13. Juni 2014 wird die Verbraucherrechterichtlinie (VRR) die derzeit geltende Richtlinie 97/7/EG zum Verbraucherschutz bei Vertragsabschlüssen im Fernabsatz (Fernabsatzrichtlinie) $^{4}$ und die gegenwärtig geltende Richtlinie 85/577/EWG zum Verbraucherschutz im Falle von außerhalb von Geschäftsräumen geschlossenen Verträgen (Haustürwiderrufsrichtlinie) ${ }^{5}$ ersetzen. Die folgenden Richtlinien werden teilweise geändert, bleiben hingegen im Übrigen in Kraft: Richtlinie 1999/44/EG zu bestimmten Aspekten des Verbrauchsgüterkaufs und der Garantien für Verbrauchsgüter ${ }^{6}$ und Richtlinie 93/13/EWG über missbräuchliche Klauseln in Verbraucherverträgen ${ }^{7}$.

Mit ihrem systematischeren Ansatz und ihrem konzeptionell vollzogenen Wechsel von einer Mindestharmonierung zur Vollharmonisierung soll die neue Verbraucherrechterichtlinie neue Maßstäbe in der Fortentwicklung des Verbraucherschutzes in der EU setzen. Es ist jedoch fraglich, ob die neue Richtlinie auch den Anforderungen und Herausforderungen eines sich auf der Höhe der Zeit befindlichen Verbraucherschutzes genügt, die sich aus dem wirtschaftlichen und technischen Fortschritt der Gegenwart und der nahen Zukunft ergeben werden.

Dieser Aufsatz wird daher versuchen, die neue Verbraucherrechterichtlinie auf Grundlage einer Untersuchung ihres Ziels und der inhaltlichen Erreichung dieses Ziels zu bewerten. Dazu werden zunächst die Entstehungsgeschichte der Richtlinie sowie ihr neuer Ansatz einer zielgerichteten Vollharmonisierung behandelt. Danach werden der Anwendungsbereich und die in der Richtlinie enthaltenen Definitionen erklärt. In systematischer Unterteilung nach den Neuerungen in den Bereichen

$1 \quad$ AB1. L 304/64 vom 22.11.2011.

2 In Deutschland hat die Bundesregierung am 19. Dezember 2012 einen Gesetzentwurf zur Umsetzung der EU- Verbraucherrechterichtlinie beschlossen, der am 6. März 2013 ins Parlament eingebracht und am 14. Juni 2013 verabschiedet worden ist (Deutscher Bundestag, 17. Wahlperiode, Drucksache 17/12637).

3 Vgl. Erwägungsgründe 4-6 VRR.

4 ABl. L 144/19 vom 4.6.1997.

5 ABl. L 372/31 vom 31.12.1985.

6 ABl. L 171/12 vom 7.7.1999.

7 ABl. L 95/29 vom 21.4.1993. 
Informationspflichten und Widerrufsrecht wird sodann der wesentliche Inhalt der Richtlinie erörtert. Schließlich wird aufgrund der vorangegangenen Analyse eine abschließende Bewertung der Richtlinie vorgenommen.

\section{Entwicklung der Verbraucherrechterichtlinie}

Die Harmonisierung des europäischen Verbraucherschutzrechts begann Anfang der 1980er Jahre. Nachdem die Ideen des US-amerikanischen Verbraucherschutzes Eingang in die Europäische Wirtschaftsgemeinschaft (EWG) gefunden hatten und sich langsam in ihren Mitgliedsstaaten entwickelten, erweiterte die Kommission ihre Prioritäten, um einen gemeinsamen Markt zu erreichen, der später zum europäischen Binnenmarkt werden sollte. Zunächst, ganz im Einklang mit der klassischen Vorstellung vom Freihandel, schien die Steigerung der Lebensqualität einzig und allein abhängig von der Förderung einer gemeinsamen europäischen Politik, die ihren Fokus auf Produktion und Warenverkehr legte. Wohlstand der Verbraucher und höherer Lebensstandard waren dabei quasi zwangsläufige Nebeneffekte des freien Marktes, sofern dieser bestimmte Bedingungen erfüllte. ${ }^{8}$ Erst mit der Einheitlichen Europäischen Akte ${ }^{9}$, die 1987 in Kraft trat, wurde das Ziel der Verwirklichung eines einheitlichen europäischen Marktes mit dem freien Verkehr von Personen, Waren, Dienstleistungen und Kapital in Art. 8a EWG (jetzt: Art. 26 Vertrag über die Arbeitsweise der Europäischen Union AEUV) aufgenommen. Der neu hinzugefügte Art. 100a EWG (jetzt: Art. 114 AEUV) gestand dem Ministerrat (jetzt: Rat der Europäischen Union) zusammen mit dem Europaparlament (EP) umfangreiche Kompetenzen zum Erlass von Maßnahmen zu, einen funktionierenden Binnenmarkt zu errichten und auszubauen. Von da an spielte Verbraucherschutzpolitik eine besondere Rolle bei der Realisierung des Binnenmarktes, und zwar in dreifachem Sinn von Entscheidungsfreiheit, legitimen Erwartungen und dem Schutz rechtlicher Interessen der Verbraucher. ${ }^{10}$

In kurzen Zeitabständen wurden mehrere Verbraucherschutzrichtlinien erlassen. Im Bereich des Verbrauchervertragsrechts sind folgende Richtlinien von Relevanz: Haustürwiderrufsrichtlinie 85/577/EWG ${ }^{11}$, Verbraucherkreditrichtlinie 87/102/ $\mathrm{EWG}^{12}$, Pauschalreiserichtlinie $90 / 314 / \mathrm{EWG}^{13}$, Klauselrichtlinie $93 / 13 / \mathrm{EWG}^{14}$, Fernabsatzrichtlinie 97/7/EG ${ }^{15}$, Verbrauchsgüterkaufrichtlinie $1999 / 44 / \mathrm{EG}^{16}$ und

8 Bourgoignie, T. Consumer Law, Common Markets, and Federalism in Europe and the United States. Berlin: De Gruyter, 1986: S. 200; Reich, N. Economic Law, Consumer Interests and EU Integration., in: Micklitz, H.-W., Reich, N., Rott, P. Understanding EU Consumer Law. Antwerp: Intersentia, 2009: S. 9.

9 ABl. L 169/1 vom 29.6.1987.

10 Reich, N.: S. 12.

11 AB1. L 372/31 vom 31.12.1985.

12 ABl. L 42/48 vom 12.2.1987.

13 ABl. L 158/59 vom 23.6.1990.

14 ABl. L 95/29 vom 21.4.1993.

15 ABl. L 144/19 vom 4.6.1997.

16 ABl. L 171/12 vom 7.7.1999. 
Dienstleistungsrichtlinie 2006/123/EG ${ }^{17}$. All diese Richtlinien folgten dem Ansatz einer Mindestharmonisierung, die zu einer Angleichung innerstaatlichen Rechts führte, indem den Mitgliedsstaaten unterschiedliche Wege der Umsetzung zugestanden wurden, sofern diese die in der Richtlinie festgelegten Mindestschutzstandards erfüllten. Zudem lag all diesen Richtlinien, die sich auf Gebiete beziehen, auf denen die Schwäche des Verbrauchers besonders ausgeprägt scheint, die Vorstellung zugrunde, dass der Verbraucher als schwächere Vertragspartei durch rechtliche Instrumente geschützt werden müsse. ${ }^{18}$ Die an für Verbraucher besonders gefährdende Situationen anknüpfenden Richtlinien über den Haustürwiderruf und missbräuchliche Vertragsklauseln sowie die wegen der erschwerten Beurteilbarkeit der Brauchbarkeit einer Leistung als besonders verbrauchergefährdend eingestufte Fernabsatzrichtlinie verfolgten hingegen auch einen situativen Schutzansatz. Schließlich folgten die Verbraucherkredit- und die Pauschalreiserichtlinie noch einem rollenspezifischen Schutzansatz, demzufolge der Verbraucher aufgrund des gefährdenden Vertragstyps gerade wegen seiner Eigenschaft als Verbraucher besonders geschützt werden muss. ${ }^{19}$

Nach Art. 114 III AEUV soll innerhalb der EU ein hoher Standard beim Verbraucherschutz gewährleistet werden. Dadurch, dass sich die EU auf Art. 114 AEUV anstelle von Art. 169 AEUV als Rechtsgrundlage für Maßnahmen des Verbraucherschutzes beruft, wird das Ziel von Kapitel 3 AEUV betont, nämlich die "Angleichung der [innerstaatlichen] ${ }^{20}$ Rechtsvorschriften" (im Bereich der Verbraucherrechte), welche innerstaatliches Recht im Endeffekt nie unberührt lässt. ${ }^{21}$ Dadurch, dass die EU in Verbraucherschutzrichtlinien aus verschiedenen Gründen vornehmlich dem Ansatz der Mindestharmonisierung gefolgt ist, variieren die EUweiten Verbraucherschutzstandards jedoch immer noch und stellen so Hindernisse im Binnenmarkt dar. Aus diesem Grund hat die Kommission eine Überprüfung des gemeinsamen Besitzstands im Verbraucherschutz ${ }^{22}$ durchgeführt, die insgesamt die folgenden acht Richtlinien umfasst: Haustürwiderrufsrichtlinie 85/577/EWG, Pauschalreiserichtlinie 90/314/EWG, Klauselrichtlinie 93/13/EWG, Teilzeitnutzungsverträgerichtlinie 94/47/EG ${ }^{23}$, Fernabsatzrichtlinie 97/7/EG, Preisangabenrichtlinie 98/6/EG ${ }^{24}$, Unterlassungsklagenrichtlinie 98/27/EG ${ }^{25}$ und Verbrauchs-

ABl. L 376/36 vom 27.12.2006.

Heininger, C-481/99 [2001] ECR I-9945, para 38; Micklitz, H.-W. The targeted full harmonisation approach: looking behind the curtain, in: Howells, G., Schulze, R. Modernising and Harmonising Consumer Contract Law. Munich: Sellier, 2009: S. 47, 83; Reich, N.: S. 46; Tonner, K., Fangerow, K. Directive 2011/83/EU on consumer rights: a new approach in European consumer law?, EUVR (2012) 2: S. 69.

Paek, K.-I. Der Bürgenschutz im Spannungsfeld zur Privatautonomie. Hamburg: Verlag Dr. , 2006: 173. Ergänzung durch den Autor.

Tonner, K., Fangerow, K.: S. 77; Twigg-Flesner, C. "Time to do the job properly" - The case for a new approach to EU consumer legislation. Journal of Consumer Policy (2010) 33: S. 357.

Grünbuch zur Überprüfung des gemeinschaftlichen Besitzstands im Verbraucherschutz, KOM (2006) 744 endg.

ABl. L 280/83 vom 29.10.1994.

ABl. L 80/27 vom 18.3.1998.

ABl. L 166/51 vom 11.6.1998. 
güterkaufrichtlinie 1999/44/EG. Eine vergleichende Analyse dieser Richtlinien resultierte in einem Verbraucherschutzkompendium ${ }^{26}$ und einer Datenbank ${ }^{27}$. Jedoch wurden die Ergebnisse dieser Analyse wie auch die Resultate der Studiengruppe zu einem Europäischen Zivilgesetzbuch sowie der Forschungsgruppe zum Privatrecht der Europäischen Gemeinschaft (Besitzstandsgruppe), die einen Entwurf eines gemeinsamen Referenzrahmens ${ }^{28}$ publiziert hatte, während der Ausarbeitung des Entwurfs der neuen Verbraucherrechterichtlinie nicht mitberücksichtigt. ${ }^{29}$ Stattdessen umfasste der letzte Entwurf der Verbraucherrechterichtlinie ${ }^{30}$ nur vier der zuvor überprüften Richtlinien, und zwar die Fernabsatzrichtlinie, die Haustürwiderrufsrichtlinie, die Klauselrichtlinie und die Verbrauchsgüterkaufrichtlinie. Schließlich wurde dieser Entwurf wiederum um die Hälfte gekürzt, so dassnurnoch dieFernabsatzrichtlinie und dieHaustürwiderrufsrichtlinie in der abschließenden Version der Verbraucherrechterichtlinie enthalten sind, die am 12. Dezember 2011 in Kraft trat.

\section{Zielgerichtete Vollharmonisierung}

Ausgangspunkt bei der Ausarbeitung der neuen Verbraucherrechterichtlinie war für den EU-Gesetzgeber die zuvor festgestellte Tatsache, dass das Potential des grenzüberschreitenden Handels weder im Fernabsatzmarkt (insbesondere im Internet) noch im Direktvertrieb (insbesondere im Dienstleistungssektor) in vollem Umfang ausgeschöpft wird, und dass die Verbraucher aus diesem Grund noch nicht ausreichend vom Binnenmarkt profitieren. ${ }^{31}$ Durch eine spürbare Verbesserung der Rechtssicherheit für Verbraucher und auch für Händler soll die unangemessene Zersplitterung des Rechts in den EU-Mitgliedsstaaten nun beendet und die Zufriedenheit der Verbraucher gestärkt werden. ${ }^{32}$ Aus diesem Grund entschied sich der EU-Gesetzgeber in der neuen Verbraucherrechterichtlinie - in Abkehr von der in den Jahren zuvor in fast allen Verbraucherschutzrichtlinien praktizierten Mindestharmonisierungspolitik - nun in den meisten Regelungsbereichen für eine maximale Harmonisierung der Verbraucherrechte. ${ }^{33}$

26 Abrufbar unter: Universität Bielefeld (Hrsg.), EG-Verbraucherrechtskompendium [interaktiv]. 2007 [aufgerufen: 2013-07-30]. <http://www.eu-consumer-law.org/consumerstudy_full_de.pdf>.

27 Abrufbar unter: Universität Bielefeld (Hrsg.), EC Consumer Law Compendium [interaktiv]. 2012 [aufgerufen: 2013-07-31]. <http://www.eu-consumer-law.org/index_de.cfm>.

28 Bar, C. von, Clive, E., Schulte-Nölke, H. (Ed.) Principles, Definitions and Model Rules of European Private Law - Draft Common Frame of Reference (DCFR), Outline Edition. Munich: Sellier, 2009.

29 Nordhausen Scholes, A. Information Requirements, in: Howells, G., Schulze, R. (Ed.) Modernising and Harmonising Consumer Contract Law. Munich: Sellier, 2009: S. 215.

30 KOM (2008) 614 endg.

31 Siehe Erwägungsgrund 5 VRR.

32 Erwägungsgründe 6-7 VRR.

33 Entsprechend den Erwägungsgründen 2 \& 13 VRR verbleibt den Mitgliedsstaaten die Kompetenz, innerstaatliche Rechtsvorschriften in Bezug zu bestimmten Aspekten der Verbraucherrechterichtlinie beizubehalten oder neu einzuführen, beispielsweise bzgl. der Anwendbarkeit der Richtlinie bei außerhalb von Geschäftsräumen geschlossenen Verträgen, bei denen die vom Verbraucher zu zahlende Gegenleistung 50 $€$ nicht überschreitet (Art. 3 IV VRR), bzgl. Informationspflichten anderer Verträge als Fernabsatz- oder 
Die Mindestharmonisierung wurde im Bereich des Verbraucherschutzes von der EU in der Vergangenheit vor allem als Kompromiss praktiziert, da Verbraucherschutzregelungen in den Mitgliedsstaaten entweder bereits existierten oder erst kürzlich übernommen worden waren, und die Mitgliedsstaaten noch nicht bereit waren, verbindliche gemeinsame Standards im Verbraucherschutz zu akzeptieren. ${ }^{34}$ Das Prinzip der Mindestharmonisierung reduzierte die Unterschiede in der innerstaatlichen Gesetzgebung dadurch, dass es einen geringeren bzw. gar keinen Schutz untersagte, während es den fortschrittlicheren Mitgliedsstaaten die Möglichkeit eröffnete, ihre höheren Verbraucherschutzstandards $\mathrm{zu}$ behalten oder für umfangreichere Schutzmaßnahmen in den anzugleichenden Rechtsbereichen zu sorgen. ${ }^{35}$ Auf diese Art und Weise wurde die durchschnittliche Qualität des Verbraucherschutzes in der EU erhöht. Auch der Europäische Gerichtshof (hiernach: EuGH) akzeptierte den Ansatz der Mindestharmonisierung in seiner Rechtsprechung, setzte seinen Mindestschutzklauseln jedoch in den Entscheidungen zur Tabakwerbung ${ }^{36}$ und zum Fall Gysbrechts ${ }^{37}$ unter Berufung auf bestimmte Aspekte des Binnenmarktes und des Verhältnismäßigkeitsgrundsatzes Grenzen. ${ }^{38}$

Vollharmonisierung war bislang nur in einigen Richtlinien neueren Datums beabsichtigt, und zwar in der Richtlinie über den Fernabsatz von Finanzdienstleistungen an Verbraucher 2002/65/EG ${ }^{39}$, in der Richtlinie über unlautere Geschäftspraktiken 2005/29/EG ${ }^{40}$, in der Verbraucherkreditrichtlinie 2008/48/EG ${ }^{41}$ und in der Teilzeitnutzungsverträgerichtlinie 2008/122/EG ${ }^{42}$. Das Prinzip der Vollharmonisierung, das mit der Verbraucherpolitischen Strategie 2002-2006 eingeführt und innerhalb der folgenden Verbraucherpolitischen Strategie 2007-2013 ${ }^{44}$ klarer ausgeführt wurde, hat nicht nur eine Angleichung, sondern eine möglichst vollständige Übereinstimmung der Verbraucherrechte in den einzelnen Mitgliedsstaaten zum Ziel. Jedoch führen selbst Vollharmonisierungsrichtlinien nicht zu gleichem Recht in allen Mitgliedsstaaten, da jeder Mitgliedsstaat den von der Richtlinie vorgegebenen Regelungsrahmen eigenverantwortlich entsprechend seiner jeweiligen Rechtstradition und den

außerhalb von Geschäftsräumen geschlossene Verträge (Art. 5 IV VRR), bzgl. vorvertraglicher Informationspflichten bei Fernabsatz- oder außerhalb von Geschäftsräumen geschlossenen Verträgen (Art. 6 VIII VRR), bzgl. der Anwendbarkeit der bei außerhalb von Geschäftsräumen geschlossenen Verträgen vorgesehenen Entgeltgrenze für Reparaturen und Instandhaltungsarbeiten von $200 €$ (Art. 7 IV VRR), oder bzgl. Regelungen, die über den Anwendungsbereich der Verbraucherrechterichtlinie hinausgehen.

34 Tonner, K., Fangerow, K.: S. 74.

35 Howells, G., Reich, N. The current limits of European harmonisation in consumer contract law. ERA Forum (2011) 12: S. 41; Reich, N.: S. 40.

36 Germany vs EP and Council, C-376/98 [2000] ECR I-8419: Paragraphen 103-105.

37 Gysbrechts, C-205/07 [2008] ECR I-9947: Paragraph 60.

38 Howells, G., Reich, N.: S. 48.

39 Abl. L 271/16 vom 9.10.2002.

40 ABl L 149/22 vom 11.6.2005.

41 ABl. L 133/66 vom 22.5.2008.

42 ABl. L 33/10 vom 3.2.2009.

43 KOM (2002) 208 endg.

44 KOM (2007) 99 endg. 
Besonderheiten des eigenen Rechtssystems ausfüllt. Somit lässt auch Vollharmonisierung stets Raum für eigenständiges innerstaatliches Recht. ${ }^{45}$

Trotz starker Kritik ${ }^{46}$ verankerte der EU-Gesetzgeber das Prinzip der Vollharmonisierung in Art. 4 VRR, wonach die Mitgliedsstaaten weder von den Bestimmungen der Verbraucherrechterichtlinie abweichende innerstaatliche Rechtsvorschriften aufrechterhalten noch einführen dürfen, soweit in der Verbraucherrechterichtlinie selbst nichts anderes bestimmt ist. Zwar mag der Ansatz der Vollharmonisierung insbesondere von klein- und mittelständischen Unternehmen ohne eigene Rechtsabteilung begrüßt werden, die über keine ausreichenden Kenntnisse über die unterschiedliche Rechtslage in den Mitgliedsstaaten der EU verfügen und deren grenzüberschreitende Handelsaktivitäten vom Volumen her so beschränkt sind, dass es sich für sie nicht lohnt, ihre Verträge, Produkte, Produktinformationen oder Verpackungen für die neuen Märkte entsprechend $\mathrm{zu}$ modifizieren. ${ }^{47}$ Jedoch muss berücksichtigt werden, dass Vollharmonisierung per se nicht unbedingt der erfolgversprechendste und am besten geeignete Weg ist, höchstmögliche Verbraucherschutzstandards zu gewährleisten und die Verbraucherzufriedenheit zu steigern. Zwar fördert eine erweiterte Umsetzung von EU-Rechtskonzepten eine "Europäisierung" des Zivilrechts in den Mitgliedsstaaten und gleicht es immer mehr an. Aber es bleibt fraglich, ob bestimmte Regelungen im Zivilrecht, wie beispielsweise Rechtsmittel bei Verletzungen, Ausschlussfristen und Benachrichtigungsfristen tatsächlich Handelshindernisse darstellen oder den Wettbewerb derart verzerren, dass eine vollständige Harmonisierung der entsprechenden Rechtsvorschriften notwendig ist. ${ }^{48}$ Denn der Ansatz der maximalen Harmonisierung bringt die Gefahr mit sich, dass minimale Standards zu Maximalstandards erhoben werden, sofern sich die Mitgliedsstaaten nicht auf höhere Verbraucherschutzstandards einigen können, was de facto zu einer EU-weiten Absenkung der Standards im Verbraucherschutz führt. So könnten z. B. in einigen Mitgliedsstaaten mit hohen Verbraucherschutzstandards einige verbraucherfreundliche Rechte oder Rechtsmittel abgeschafft oder neue Hürden für den Zugang zu den Gerichten aufgestellt werden, wenn die neu gesetzte Vollharmonisierung nur soweit geht wie die bisherige Mindestharmonisierung. ${ }^{49}$ So würden einige Mitgliedsstaaten nämlich ihrer vorher

45 Tonner, K., Fangerow, K.: S. 75.

46 Siehe beispielhaft für die gegen den Ansatz der Vollharmonisierung in diesem Bereich gerichteten Kritiken: Faure, M. Towards a Maximum Harmonization of Consumer Contract Law?!?, Maastricht Journal, 2008/4: S. 440 f.; Micklitz, H.-W., Reich, N. Crónica de una muerte anunciada: The Commission Proposal for a 'Directive on Consumer Rights', CMLRev, 2009: S. 471; Rott, P., Terryn E. The proposal for a Directive on consumer rights: no single set of rules. ZEuP, 2009: S. 456; Smits, J. M. Full Harmonization of Consumer Law? A Critique on the Draft Directive on Consumer Rights. European Review of Private Law, 2010, Vol. 18: S. 5-14; Tonner, K., Tamm, M. Der Vorschlag für eine Richtlinie über Verbraucherrechte und seine Auswirkungen auf das nationale Verbraucherrecht. JZ, 2009: S. 277; Twigg-Flesner, C., Metcalfe, D. The Proposed Consumer Rights Directive - less haste, more thought?, European Review of Contract Law, 2009: S. 368; Wilhelmsson, T. Full harmonisation of consumer law?, ZEuP, 2008: S. 225.

47 Howells, G., Schulze, R. Overview of the Proposed Consumer Rights Directive, in: Howells, G., Schulze, R. (Ed.) Modernising and Harmonising Consumer Contact Law. Munich: Sellier, 2009: S. 6.

49 Z. B. erlischt in Deutschland gem. § 355 III S. 3 BGB das Widerrufsrecht bei Verbraucherverträgen nicht, 
durch den Mindestharmonisierungsansatz garantierten Freiheit beraubt, über die festgeschriebenen Mindeststandards hinaus einen besseren Schutz der Verbraucher zu gewährleisten. In diesem Fall würde der Vollharmonisierungsansatz der Verbraucherrechterichtlinie das Vertrauen der Verbraucher eher enttäuschen als verbessern.

Um ein Absinken der Verbraucherschutzstandards zu vermeiden, vollzieht die Verbraucherrechterichtlinie keine absolute Vollharmonisierung. Dadurch, dass einige Regelungsbereiche, wie z. B. zusätzliche vorvertragliche Informationspflichten für Fernabsatz-, außerhalb von Geschäftsräumen geschlossene und andere Verträge explizit in der Regelungskompetenz der Mitgliedsstaaten verbleiben, und dadurch, dass die Rechtsfolgen bei Nichterfüllung der vorvertraglichen Informationspflichten - mit Ausnahme der Ausweitung des Widerrufsrechts-ausschließlich derRegelungskompetenz der Mitgliedsstaaten unterliegen, folgt die Verbraucherrechterichtlinie eher dem Prinzip einerzielgerichteten Vollharmonisierung. Dies bedeutet, dass sichdie Vollharmonisierung nicht auf alle Bereiche der Richtlinie erstreckt, sondern den Mitgliedsstaaten die Freiheit lässt, selbstständig Rechtsvorschriften für regelungsbedürftige Problembereiche beizubehalten oder einzuführen, welche nicht von der Richtlinie umfasst sind bzw. von deren Regelungszweck nicht gedeckt sind. ${ }^{50}$ Dieser Ansatz steht auch im Einklang mit der geteilten Zuständigkeit der EU und ihrer Mitgliedsstaaten im Bereich des Verbraucherschutzes gem. Art. 4 II f AEUV sowie den Grundsätzen der loyalen Zusammenarbeit (Art. 4 III EUV) und Subsidiarität (Art. 5 III EUV).

\section{Anwendungsbereich der Verbraucherrechterichtlinie}

Der ursprüngliche Entwurf der Verbraucherrechterichtlinie hatte eigentlich die Fernabsatzrichtlinie, die Haustürwiderrufsrichtlinie, die Verbrauchsgüterkaufrichtlinie und die Klauselrichtlinie umfassen sollen. ${ }^{51}$ Jedoch wurde von dieser Intention während des Gesetzgebungsverfahrens immer mehr abgewichen. So blieb schließlich eine Verbraucherrechterichtlinie übrig, in der lediglich die alte Fernabsatzrichtlinie und

wenn der Verbraucher zuvor nicht ordnungsgemäß über sein Widerrufsrecht informiert worden ist. Dieser Paragraph wurde ins deutsche BGB aufgenommen, nachdem der EuGH im Fall 481/99 (Heininger/Bayerische Hypo- und Vereinsbank $A G$ ) ECR 2001 I-09945 entschieden hatte, dass sich aus der Haustürwiderrufsrichtlinie ein unbefristetes Widerrufsrecht ergibt. Nach der Entscheidung des EuGH gewährte der deutsche Gesetzgeber dieses unbefristete Widerrufsrecht jedoch nicht nur bei Haustürgeschäften, sondern für alle Verbraucherverträge, bei denen ein Widerrufsrecht besteht, wie beispielsweise bei Verbraucherkredit- oder Fernabsatzverträgen. Gemäß Art. 10 I VRR endet das Widerrufsrecht künftig 12 Monate nach Ablauf der ursprünglichen Widerrufsfrist, wenn der Händler den Verbraucher nicht über dessen Widerrufsrecht belehrt hat. In Umsetzung der auf dem Prinzip der Vollharmonisierung basierenden Verbraucherrechterichtlinie ist der deutsche Gesetzgeber nun gezwungen, § 355 III S. 3 BGB wieder abzuschaffen, da er den Verbrauchern weitergehende Rechte einräumt als in der Verbraucherrechterichtlinie als maximales Recht vorgegeben. Dies bedeutet eine klare Einschränkung des bis dahin umfangreicheren Verbraucherschutzes.

50 Tonner, K.Fangerow, K.: S. 78.

51 KOM (2008) 614 endg. 
die Haustürwiderrufsrichtlinie enthalten sind, zuzüglich einiger Vorschriften über vorvertragliche Informationspflichten.

Gemäß Art. 1 I VRR ist die Richtlinie "auf Verträge, die zwischen Verbrauchern und Unternehmern geschlossen werden," anwendbar, einschließlich solcher Verträge, die außerhalb von Geschäftsräumen und solchen, die unter Verwendung von Fernkommunikationsmitteln geschlossen werden. Darüber hinaus ist sie anwendbar auf Verträge, die die Lieferung von Wasser, Gas, Strom oder Fernwärme zum Gegenstand haben. Jedoch fallen eine Reihe anderer in Art. 1 III VRR aufgelisteter Verträge nicht in den Anwendungsbereich der Verbraucherrechterichtlinie, darunter Verträge über soziale Dienstleistungen, Gesundheitsdienstleistungen, Glücksspiele, Finanzdienstleistungen, Eigentumserwerb oder -übertragung an Immobilien, Wohnraumvermietung, Bau von Gebäuden, Lieferung von Lebensmitteln und Haushaltsgegenständen des täglichen Bedarfs sowie Personenbeförderung.

\section{In der Verbraucherrechterichtlinie enthaltene Begriffsbestimmungen}

Entsprechend dem Zweck der Richtlinie sind die wichtigsten Begriffe und Vertragstypen in Art. 2 VRR definiert: Gemäß Art. 2 I VRR ist ein Verbraucher ,jede natürliche Person, die [...] zu Zwecken handelt, die außerhalb ihrer gewerblichen, geschäftlichen, handwerklichen oder beruflichen Tätigkeit liegen “52. Hierbei verwendet die Richtlinie die allgemeine Kerndefinition des Verbraucherbegriffs, die sich in ähnlichem Wortlaut auch in anderen EU-Richtlinien zum Verbraucherschutz findet. Damit versäumt es die Richtlinie jedoch, zusätzlich noch speziellere Verbraucherbegriffe in bestimmten Bereichen ausdrücklich mit aufzunehmen, die ebenfalls von der neuen Verbraucherrechterichtlinie umfasst sind, wie beispielsweise in den Bereichen Telekommunikation und Energie. Insbesondere im Bereich Telekommunikation, der u. a. die Nutzung von Handys, Smartphones und Internet umfasst, wird anstelle des Begriffs "Verbraucher" im allgemeinen Sprachgebrauch der Begriff "Nutzer" verwendet ebenso wie der Begriff „Kunde“ im Energiesektor. ${ }^{53}$

Das vertragliche Pendant zum Verbraucher, der Unternehmer, wird in Art. 2 II VRR als jede natürliche oder juristische Person definiert, die ,zu Zwecken tätig wird, die ihrer gewerblichen, geschäftlichen, handwerklichen oder beruflichen Tätigkeit zugerechnet werden können" 54 .

Die Verträge, die von der Verbraucherrechterichtlinie umfasst sind, können entweder Kaufverträge, Fernabsatzverträge, außerhalb von Geschäftsräumen abgeschlossene Verträge oder auch akzessorische Verträge sein. Gemäß Art. 2 V VRR

52 Art. 2 I VRR.

53 Siehe Rahmenrichtlinie über einen gemeinsamen Rechtsrahmen für elektronische Kommunikationsnetze und -dienste (Abl. L 108/33 vom 24.04.2002), Elektrizitätsbinnenmarktrichtlinie 2009/72/EG (ABl. L 211/55 vom 14.08.2009) und Erdgasbinnenmarktrichtlinie 2009/73/EG (ABl. L 211/94 vom 14.08.2009).

54 Art. 2 II VRR. 
ist ein Kaufvertrag jeder Vertrag, durch den der Unternehmer das Eigentum an Waren an den Verbraucher überträgt, der seinerseits dafür den Kaufpreis zahlt oder zusagt. Im Gegensatz dazu ist ein Dienstleistungsvertrag ein Vertrag, bei dem der Verbraucher für eine vom Unternehmer an ihn erbrachte oder zugesagte Dienstleistung einen Preis zahlt (Art. 2 VI VRR). Ein Fernabsatzvertrag nach Art. 2 VII VRR ist im weiten Sinne jeder Vertrag, der zwischen Unternehmer und Verbraucher unter ausschließlicher Verwendung von Fernkommunikationsmitteln wie Internet, Telefon oder Fax geschlossen wird, ohne dass beide Vertragsparteien gleichzeitig bei Vertragsschluss körperlich anwesend sind. Ein außerhalb von Geschäftsräumen abgeschlossener Vertrag ist hingegen ein Vertrag zwischen Unternehmer und Verbraucher, der an einem Ort geschlossen wird, der nicht zu den Geschäftsräumen des Unternehmers zählt (Art. 2 VIII VRR). Gemäß Art. 2 XV VRR ist ein akzessorischer Vertrag im Sinne der Richtlinie ein Vertrag, bei dem ein Dritter im Zusammenhang mit einem Fernabsatzvertrag oder einem außerhalb von Geschäftsräumen geschlossenen Vertrag als Vermittler beim Erwerb von Waren oder Dienstleistungen durch den Verbraucher vom Unternehmer oder einem Dritten beteiligt ist.

Im Bewusstsein der Problematik der sog. Verträge mit doppeltem Zweck, die nicht eindeutig von Verträgen, die für gewerbliche oder geschäftliche Zwecke geschlossen werden, abgegrenzt werden können, weil sie gleichzeitig sowohl dem beruflichen als auch dem privaten Bereich mindestens einer der Vertragsparteien zugeordnet werden können, legte der EU-Gesetzgeber in der Verbraucherrechterichtlinie einige Auslegungsrichtlinien fest. Der engen Auslegung des EuGH im Fall Johann Gruber ${ }^{55}$ folgend, enthält Erwägungsgrund 17 VRR die Festlegung, dass im Fall eines Vertrages mit doppeltem Zweck, in dem der "gewerbliche Zweck im Gesamtzusammenhang des Vertrags nicht überwiegend [ist]", [...] diese Person auch als Verbraucher betrachtet werden [soll]“ "56. Wie der Wortlaut nahelegt, soll bei Verträgen mit doppeltem Zweck wohl im Zweifel die Verbrauchereigenschaft angenommen werden, so dass ein überwiegend gewerblicher Zweck erst bewiesen werden muss. ${ }^{57}$

\section{Wesentlicher Inhalt der Verbraucherrechterichtlinie}

Neben der Sicherstellung eines hohen Harmonisierungsgrades durch die Umsetzung des Prinzips der zielgerichteten Vollharmonisierung bringt die Verbraucherrechterichtlinie auch in den Bereichen der Informationspflichten und des Widerrufsrechts erhebliche Weiterentwicklungen der bestehenden Rechtslage mit sich. Diese neuen Informationspflichten und die geänderten Bestimmungen zum Widerrufsrecht ersetzen die derzeit noch gültige Fernabsatzrichtlinie und Haustürwiderrufsrichtlinie, die formell am 13. Juni 2014 aufgehoben werden (Art. 31 VRR). Die Kernpunkte der in der Verbraucherrechterichtlinie enthaltenen und für den Schutz der Verbraucher relevanten Neuerungen werden im Folgenden überblicksartig erörtert:

55 Siehe Johann Gruber vs Bay Wa AG, C-464/01[2005] ECR 2005 I-00439.

56 Erwägungsgrund 17 VRR.

57 Grundmann, S. Die EU-Verbraucherrechte-Richtlinie, JZ, 2013: S. 55. 


\section{1) Informationspflichten}

Die Verbraucherrechterichtlinie folgt dem Informationsansatz, der bislang auch die Grundlage aller früheren vertragsbezogenen EU-Verbraucherschutzrichtlinien war..$^{58}$ Dieser geht von der Prämisse aus, dass ein Verbraucher, dem alle verfügbaren Informationen zugänglich gemacht werden, in Abwägung aller Umstände eine informierte und wohlüberlegte Entscheidung treffen wird. Gleichzeitig wird davon ausgegangen, dass ein informierter Verbraucher auch ein zufriedener Verbraucher ist, der fähig und willens ist, seine Auswahl zum Erwerb von Waren und Dienstleistungen auf dem EU-Binnenmarkt unabhängig von Staatsgrenzen zu treffen. ${ }^{59} \mathrm{Um}$ dieses Ziel zu erreichen, legt die Verbraucherrechterichtlinie mehrere vorvertragliche Informationspflichten fest, die jeder Unternehmer erfüllen muss, um den Verbraucher besser zu schützen und diesen in die Lage zu versetzen, alle relevanten Tatsachen bei seiner Entscheidung zu berücksichtigen, bevor er einen Vertrag schließt. Diese in der Verbraucherrechterichtlinie enthaltenen vorvertraglichen Informationspflichten lassen sich systematisch wie folgt zusammenfassen:

a) Produkt- und Dienstleistungsinformationen

Art. 5 und 6 VRR enthalten vorgeschriebene Informationen bzgl. der angebotenen Ware oder Dienstleistung des Unternehmers oder des Unternehmens, die der Unternehmer dem Verbraucher mitzuteilen hat, bevor der Vertrag zwischen beiden geschlossen wird. Die im Einzelnen erforderlichen Informationen sind dabei vom Vertragstyp abhängig, d. h. davon, ob es sich beim zu schließenden Vertrag um einen Fernabsatzvertrag oder um einen außerhalb von Geschäftsräumen abzuschließenden Vertrag handelt. Ungeachtet des Vertragstyps müssen alle Informationen in klarer und verständlicher Weise mitgeteilt werden. Bei Fernabsatzverträgen und außerhalb von Geschäftsräumen abgeschlossenen Verträgen gelten wegen der besonderen Art und Weise des Vertragsschlusses erhöhte Informationsanforderungen.

Art. 5 VRR enthält eine Auflistung von Informationspflichten für andere Verträge als Fernabsatzverträge und außerhalb von Geschäftsräumen abgeschlossene Verträge, die nicht abschließend ist und die beispielsweise Informationspflichten bzgl. der wesentlichen Eigenschaften der Ware oder Dienstleistung, der Identität des Unternehmers, des Gesamtpreises der Waren und Dienstleistungen inklusive Steuern sowie Fracht- und Versandkosten umfasst. Der Umstand, dass die Auflistung der Informationspflichten gem. Art. 5 IV VRR ausdrücklich nicht abschließend ist, stellt eine Ausnahme vom Prinzip der Vollharmonisierung dar und ermächtigt die Mitgliedsstaaten, zusätzliche vorvertragliche Informationspflichten einzuführen oder aufrechtzuerhalten.

Art. 6 VRR erweitert die vorvertraglichen Informationspflichten für Unternehmer bei Fernabsatzverträgen und außerhalb von Geschäftsräumen abgeschlossenen Verträgen auf insgesamt $20 .{ }^{60}$ Neben den wesentlichen Eigenschaften der Ware oder Dienstleistung,

58 Vgl. Reich, N.: S. 21 ff., 45 f.

59 Nordhausen Scholes, A.: S. 216.

60 Im Vergleich dazu enthält die Fernabsatzrichtlinie in ihrem Art. 4 I insgesamt nur neun verschiedene vor- 
der Identität des Unternehmers, dem Gesamtpreis der Waren und Dienstleistungen inklusive Steuern, Fracht- und Versandkosten muss der Unternehmer den Verbraucher u. a. zusätzlich noch über seine Anschrift bzw. Geschäftsanschrift, die Kosten der für den Einsatz für den Vertragsabschluss benutzen Fernkommunikationstechnik, die Einzelheiten der Ausübung eines bestehenden Widerrufsrechts sowie über verschiedene technische Details des Vertrags oder der erworbenen Ware bzw. Dienstleistung, wie z. B. die Interoperabilität digitaler Inhalte mit vom Verbraucher genutzter Hard- und Software, aufklären. Darüber hinaus legen Art. 7 und 8 VRR formale (Informations-) Anforderungen für außerhalb von Geschäftsräumen abgeschlossene Verträge (Art. 7 VRR) und Fernabsatzverträge (Art. 8 VRR) fest. Einerseits stellt die Auflistung von insgesamt 20 Informationspflichten des Unternehmers bei Fernabsatzverträgen und außerhalb von Geschäftsräumen abgeschlossenen Verträgen - bei gleichzeitiger Möglichkeit des Erlasses zusätzlicher Informationspflichten für die Mitgliedsstaaten ${ }^{61}$ - sicher, dass dem Verbraucher vor Vertragsschluss alle notwendigen Informationen mitgeteilt worden sind. Andererseits birgt diese große Anzahl von Informationen, die der Verbraucher lesen und auch noch verstehen muss, die Gefahr, diesen zu verwirren und ihn davon abzuschrecken bzw. abzuhalten, die für ihn relevanten Informationen zu finden und sorgfältig auf ihren Inhalt hin zu überprüfen. ${ }^{62}$

\section{b) Preistransparenz}

Für jeden von der Verbraucherrechterichtlinie umfassten Vertragstyp müssen Unternehmer gem. Art. $5 \mathrm{I}$ c und $6 \mathrm{I}$ e VRR den Gesamtpreis der Waren oder Dienstleistungen wie auch die etwaigen Zusatzkosten angeben. Dadurch werden insbesondere die Rechte der Verbraucher bei Online-Käufen gestärkt, die fortan keine „versteckten“ zusätzlichen Gebühren oder sonstigen Kosten mehr entrichten müssen, über die sie nicht ausdrücklich und in verständlicher Weise informiert worden sind, bevor sie ihre Bestellung aufgegeben haben.

\section{c) Klare Information über Rücksendekosten im Falle des Widerrufs}

In den Fällen, in denen der Händler die Kosten für die Rücksendung der Ware auf den Verbraucher abwälzen will, nachdem dieser den Vertrag widerrufen hat, muss er den Verbraucher vor Vertragsschluss darüber informieren. Wenn er den Verbraucher nicht darüber informiert hat, hat der Händler die Rücksendekosten selbst zu tragen (Art. 6 I h, 14 I 3 VRR). Entsprechend dem Erwägungsgrund 36 VRR müssen Händler bei OnlineVerkäufen oder anderen Arten von Fernabsatzverträgen bereits vor Vertragsschluss eine

vertragliche Informationspflichten, wohingegen die Haustürwiderrufsrichtlinie keine Informationspflichten des Unternehmers separat aufführt. Lediglich nach Art. 4 Haustürwiderrufsrichtlinie ist der Unternehmer verpflichtet, "den Verbraucher [...] schriftlich über sein Widerrufsrecht innerhalb der in Artikel 5 festgelegten Fristen [diese richten sich nach dem Zeitpunkt der Widerrufsbelehrung]* zu informieren und dabei den Namen und die Anschrift einer Person anzugeben, der gegenüber das Widerrufsrecht ausgeübt werden kann". *Ergänzung durch den Autor.

61 Art. 6 VIII 1 VRR.

62 Wendlandt, B. EC Directives for Self-Employed Commercial Agents and on Time-Sharing - Apples, Oranges and the Core of the Information Overload Problem, in: Howells, G., Janssen, A., Schulze, R. (Ed.) Information Rights and Obligations. Aldershot: Ashgate Publishing, 2005: S. 74 ff. 
vernünftige Schätzung der Höchstkosten angeben, die für die Rücksendung sperriger Waren wie Möbeln anfallen können.

d) Beseitigung versteckter Gebühren und Kosten im Internet

Die Verbraucherrechterichtlinie schützt Verbraucher auch vor sog. Kostenfallen im Internet. Damit sind die Fälle gemeint, in denen betrügerische Anbieter durch Verschleierung der Kostenpflichtigkeit von als kostenlos deklarierten oder als unentgeltlich zu erwartenden Leistungen wie Horoskopen, Wettervorhersagen, Rezepten oder allen Arten von Downloads den arglosen Verbraucher zur unbewussten Entrichtung eines Entgelts verleiten. Gemäß Art. 8 II VRR besteht die Zahlungsverpflichtung des Verbrauchers erst, nachdem er ausdrücklich bestätigt hat, von der Kostenpflichtigkeit der Bestellung Kenntnis genommen zu haben.

e) Verbot der Voreinstellung für Zusatzleistungen bei OnlineBestellungen

Gegenwärtig sind Verbraucher bei Bestellungen und Buchungen im Internet, die kostenpflichtige Zusatzleistungen wie Reiseversicherungen oder Servicepakete anbieten, häufig noch gezwungen, die bereits durch die webseitigen Voreinstellungen des Anbieters vorgenommene Buchung der Zusatzleistung durch Deaktivierung eines automatisch ausgewählten Feldes rückgängig zu machen. Art. 22 VRR verbietet diese kostenpflichtigen Zusatzleistungen bei Online-Geschäften nun, wenn der Unternehmer entsprechende Voreinstellungen verwendet und keine ausdrückliche Zustimmung des Verbrauchers zur Hinzubuchung der Zusatzleistung eingeholt hat.

\section{f) Informationen über digitale Inhalte}

Informationen über digitale Inhalte, insbesondere die Interoperabilität mit Hardware und Software (Art. 5 I g, 6 I s VRR) und die Anwendbarkeit technischer Schutzmaßnahmen für solche Inhalte (Art. 5 I h, 6 I r VRR) betreffend, müssen nun klarer und verständlicher sein. Dies betrifft beispielsweise die Einschränkung des Rechts des Verbrauchers zur Vervielfältigung.

\section{g) Keine Zusatzkosten bei Kreditkartenzahlung}

Art. 19 VRR verbietet es Unternehmern, Verbrauchern für irgendeine der angebotenen Zahlungsarten (insbesondere Zahlung per Kreditkarte) mehr zu berechnen, als es den Unternehmer tatsächlich kostet, Zahlungen in der bestimmten Zahlungsart zu akzeptieren.

h) Keine unangemessenen Kosten für Hotlines

Unternehmern, die Verbrauchern Telefon-Hotlines für die direkte Kontaktaufnahme bei Fragen zum Vertrag anbieten, ist es gem. Art. 21 VRR untersagt, für den Anruf bei der Hotline höhere Gebühren zu verlangen als bei der Nutzung des normalen Festnetzbzw. Handytarifs anfallen. 


\section{2.) Widerrufsrecht}

Zweck des Widerrufsrechts ist der Schutz des Verbrauchers vor schnellen und unüberlegten Entscheidungen. ${ }^{63}$ Innerhalb einer relativ kurz bemessenen Widerrufsfrist soll der Verbraucher noch einmal seine Entscheidung zum Vertragsschluss überdenken können, in einigen Fällen selbst dann, wenn der Vertrag bereits von beiden Vertragsparteien erfüllt worden ist. Die Verbraucherrechterichtlinie stärkt damit die Rechte der Verbraucher auch in den Fällen eines Widerrufs und legt deren jeweilige Rechte und Pflichten fest, ohne dabei die Interessen des Unternehmers außer Acht zu lassen.

\section{a) Verlängerung der Widerrufsfrist}

Gemäß Art. 9 I VRR wird die gegenwärtig geltende 7-Tage-Frist, innerhalb welcher der Verbraucher einen Fernabsatzvertrag oder einen außerhalb von Geschäftsräumen geschlossenen Vertrag widerrufen kann, auf 14 Kalendertage ausgedehnt. Bei Dienstleistungsverträgen beginnt diese Widerrufsfrist am Tag des Vertragsschlusses (Art. 9 II a), bei Kaufverträgen an dem Tag, an dem der Verbraucher oder ein von ihm benannter Dritter in den Besitz der Ware gelangt ist (Art. 9 II b). Unter bestimmten Voraussetzungen verlängert sich die Widerrufsfrist, beispielsweise um bis zu 12 Monate, wenn der Unternehmer den Verbraucher nicht frist- und ordnungsgemäß über sein Widerrufsrecht belehrt hat (Art. 10 I VRR). Sie verlängert sich hingegen um 14 Tage, wenn der Unternehmer dem Verbraucher die erforderliche Widerrufsbelehrung binnen 12 Monaten ab dem beim jeweiligen Vertragstyp für die Widerrufsfrist maßgeblichen Tag erteilt hat (Art. 10 II VRR). Das Widerrufsrecht besteht auch unter anderen Voraussetzungen länger, z. B. bei auf Bitten des Verbrauchers erfolgten Besuchen oder Online-Auktionen, wenn der Vertragspartner des Verbrauchers ein professioneller Händler bzw. Unternehmer ist.

Ausnahmen vom Widerrufsrecht sind in Art. 16 VRR aufgelistet, darunter beispielsweise für Dienstleistungsverträge, bei denen die Dienstleistung bereits vollständig erbracht worden ist (z. B. Downloads von Musik oder Videos aus dem Internet bis zu dem Zeitpunkt, in dem der Vorgang des Herunterladens beginnt), für Verträge zur Lieferung von Waren, die nach Kundenspezifikation gefertigt, auf die persönlichen Bedürfnisse des Kunden zugeschnitten oder für Verträge über verderbliche Waren oder solche, deren Verfallsdatum schnell überschritten werden würde (z. B. Nahrungsmittel).

b) Einführung eines EU-weiten Muster-Widerrufsformulars

Wenn ein Verbraucher seinen bereits geschlossenen Fernabsatz- oder außerhalb von Geschäftsräumen geschlossenen Vertrag widerrufen will, kann er künftig gem. Art 11 I VRR ein standardisiertes Muster-Widerrufsformular benutzen, das in Anhang I (B) des VRR enthalten ist. Ungeachtet dessen ist der Verbraucher entsprechend

63 Loos, M. Rights of Withdrawal, in: Howells, G., Schulze, R. (Ed.) Modernising and Harmonising Consumer Contract Law. Munich: Sellier, 2009: S. 239. 
Erwägungsgrund 44 VRR weiterhin dazu berechtigt, den Widerruf mit seinen eigenen Worten und unter Nutzung jeglicher Art von Kommunikationsmitteln zu erklären.

c) Umfangreichere Rechte bei Rückzahlung

Um die Abwicklung des Widerrufs zu beschleunigen, verpflichtet Art. 13 I VRR den Unternehmer, dem Verbraucher alle von diesem erhaltenen Zahlungen einschließlich der Versandkosten - zu erstatten, und zwar innerhalb einer Frist von 14 Tagen ab Kenntnis des Widerrufs. Im Gegenzug ist der Verbraucher gem. Art. 14 I VRR dazu verpflichtet, dem Unternehmer die empfangenen Waren zurückzugeben bzw. zurückzusenden, und zwar auch nicht später als innerhalb einer Frist von 14 Tagen ab der Erklärung seines Widerrufs. Um die Rückgewähr der Vertragsleistungen Zug um Zug sicherzustellen, gibt Art. 13 III VRR dem Unternehmer das Recht, "die Rückzahlung [zu] verweigern, bis er die Waren wieder zurückerhalten hat oder bis der Verbraucher den Nachweis erbracht hat, dass er die Waren zurückgeschickt hat". Grundsätzlich trägt der Unternehmer dabei das Risiko für einen Verlust oder eine Beschädigung der Waren beim Transport, solange der Verbraucher die Waren nicht in Besitz hat (Art. 20 VRR).

\section{Fazit}

Das Rechtsgebiet des EU-Verbraucherschutzrechts ist gegenwärtig eine bunte Mischung aus Rechtsnormen, die ihre Grundlage entweder im EU-Recht oder im innerstaatlichen Recht der Mitgliedsstaaten haben. In den Bereichen, in denen der EUGesetzgeber in Form von Richtlinien aktiv geworden war, haben die Mitgliedsstaaten zumindest gemeinsame Mindeststandards im Verbraucherschutz erreicht. In den Bereichen, in denen der EU-Gesetzgeber bislang untätig geblieben war, waren die Mitgliedsstaaten in der Lage, diese Lücke mit ihren eigenen innerstaatlichen Regelungen zum Verbraucherschutz auszufüllen. Unter Berücksichtigung der Fernabsatzrichtlinie und der Haustürwiderrufsrichtlinie, die ab dem 13. Juni 2014 durch die neue Verbraucherrechterichtlinie aufgehoben werden, existieren nach dem EU-Beitritt Kroatiens zurzeit 28 nationale Regelungen zu Fernabsatz- und Haustürgeschäfteverträgen. Vor dem Hintergrund dieser gegenwärtigen Rechtslage ist der zielgerichtete Vollharmonisierungsansatz der Verbraucherrechterichtlinie generell zu begrüßen.

Dem Prinzip der zielgerichteten Vollharmonisierung folgend, überlässt die Verbraucherrechterichtlinie ausdrücklich nur einige Bereiche, wie z. B. die Festlegung zusätzlicher vorvertraglicher Informationspflichten für Fernabsatzverträge, außerhalb von Geschäftsräumen geschlossene sowie andere Verträge, der weiteren Regelungskompetenz der Mitgliedsstaaten. Dadurch, dass die Regelung der Rechtsfolgen einer Verletzung der vorvertraglichen Informationspflichten - mit Ausnahme der Verlängerung der Widerrufsfrist in diesen Fällen - weiterhin Sache der Mitgliedsstaaten bleibt, verfehlt die Verbraucherrechterichtlinie jedoch ihr Ziel einer Vollharmonisierung. Denn, wenn eine Verletzung der Pflicht, den Verbraucher vor Vertragsschluss umfassend 
zu informieren und über sein Widerrufsrecht aufzuklären, in einem Mitgliedsstaat einen Schadensersatzanspruch gegen den Unternehmer zu begründen vermag, in einem anderen Mitgliedsstaat aber die Nichtigkeit des gesamten Vertrages zur Folge haben kann, dann wird die Kluft verschiedener Verbraucherschutzstandards in den Mitgliedsstaaten durch die Verbraucherrechterichtlinie eher vergrößert als verkleinert. ${ }^{64}$ Darüber hinaus, wie bereits oben dargelegt, führt der zielgerichtete Vollharmonisierungsansatz zugunsten der Schaffung einheitlicher Standards und einer verbesserten Rechtssicherheit für Unternehmer in einigen Fällen sogar zu einem Absenken der Verbraucherschutzstandards in einigen Mitgliedsstaaten.

Unstrittig wird die Situation der Verbraucher im Bereich der Fernabsatzverträge und der außerhalb von Geschäftsräumen geschlossenen Verträge (früher: Haustürgeschäfte) durch die neue Verbraucherrechterichtlinie verbessert, insbesondere hinsichtlich der Rechte der Verbraucher auf Information und zum Widerruf. Zum einen versetzt sie den gut informierten Verbraucher in die Lage, eine wohl überlegte Entscheidung dergestalt zu treffen, ob er eine Ware oder Dienstleistung von einem bestimmten Unternehmer erwerben will. Zum anderen verlängert sie die Widerrufsfrist und legt die Rechte und Pflichten beider Vertragsparteien im Falle des Widerrufs des Vertrages durch den Verbraucher eindeutig fest.

Aus diesen Gründen sollte man die neue Verbraucherrechterichtlinie weder unterschätzen noch überbewerten. Einerseits vereinigt sie lediglich zwei alte Richtlinien unter dem Dach einer neuen und verpasst dabei die Gelegenheit, auch noch weitere existierende Richtlinien miteinzubeziehen, die ebenfalls Gegenstand einer umfassenden Überprüfung waren. Beispielsweise hätte die Verbraucherrechterichtlinie auch noch eine Auflistung von missbräuchlichen Klauseln in Verbraucherverträgen aufnehmen oder eine einheitliche Widerrufs- bzw. Rücktrittsfrist für alle Arten von Verbraucherverträgen festlegen können. Andererseits kann der Verbraucherrechterichtlinie mit ihrem vollzogenen Schritt, vormals auf mehrere Rechtsquellen verstreute Regelungen zu vereinfachen und mittels einer Richtlinie in einem Rechtsgebiet zusammenzufassen, Modellcharakter für künftige Gesetzgebungsvorhaben auf EU-Ebene zukommen. In diesem Sinne könnte die Verbraucherrechterichtlinie ein erster und wesentlicher Schritt zu einem gemeinsamen europäischen Vertragsrecht (zumindest für privatrechtliche und grenzüberschreitende Geschäfte) sein, egal ob dieses Vorhaben durch eine Änderung der Verträge, durch eine Verordnung oder weiterhin durch (Vollharmonisierungs-) Richtlinien umgesetzt werden soll. Nur durch die Gewährleistung eines für alle Mitgliedsstaaten einheitlichen und allgemein verbindlichen Fundus von Verbraucherrechten wird der Verbraucher tatsächlich ins Zentrum des Binnenmarktes gerückt, wie es vom Europaparlament und Rat in deren Entwurf zur Regelung des

64 Siehe auch: Grynbaum, L. Precontractual information duties: the foreseeable failure of full harmonisation, in: Schulte-Nölke, H., Tichy, L. (Ed.) Perspectives for European Consumer Law. Towards a Directive on consumer rights. Munich: Sellier, 2010: S. 11; Gsell, B., Schellhase, H. M. Vollharmonisiertes Verbraucherkreditrecht - ein Vorbild für die weitere europäische Angleichung des Verbrauchervertragsrechts?, JZ, 2009: S. 23 f.; Loos, M. Full harmonisation as a regulatory concept and its consequences for the national legal orders. The example of the Consumer rights directive, in: Stürner, M. (Ed.) Vollharmonisierung im Europäischen Verbraucherrecht? München: Sellier, 2010: S. 67; Rott, P., Terryn, E.: S. 459. 
Verbraucherprogramms 2014-202065 als grundlegendes Ziel formuliert worden ist. Darin genanntes Ziel ist es, dass der Verbraucher keine Zurückhaltung mehr zeigt, auch grenzüberschreitend Fernabsatzgeschäfte zu tätigen. Es bleibt allerdings fraglich, ob irgendwelche Harmonisierungsmaßnahmen des EU-Rechts tatsächlich in der Lage sein werden, das Volumen grenzüberschreitender Geschäfte dem innerstaatlicher Geschäfte anzunähern. Sind für viele Verbraucher und Händler doch unterschiedliche Sprache, kulturelle Einstellungen und Gepflogenheiten, praktische Bestimmungen zur Kennzeichnung und Verpackung von Waren, technische Standards oder die fragliche gerichtliche Durchsetzbarkeit etwaiger Ansprüche weitaus größere Hindernisse für die Teilnahme am grenzüberschreitenden Handel und Geschäftsverkehr als das in den EUStaaten derzeit teilweise noch uneinheitliche Verbrauchervertragsrecht.

\section{Literaturverzeichnis}

Bar, C. von; Clive, E.; Schulte-Nölke, H. (Ed.) Principles, Definitions and Model Rules of European Private Law - Draft Common Frame of Reference (DCFR), Outline Edition. Munich: Sellier, 2009.

Bourgoignie, T. Consumer Law, Common Markets and Federalism in Europe and the United States. Berlin: de Gruyter, 1986.

Faure, M. Towards a Maximum Harmonization of Consumer Contract Law?!? Maastricht Journal, 2008/4: 433-445.

Grundmann, S. Die EU-VerbraucherrechteRichtlinie.JZ, 2013: 53-65.

Grynbaum, L. Precontractual information duties: the foreseeable failure of full harmonisation. In Schulte-Nölke, H.; Tichy, L. (Ed.) Perspectives for European Consumer Law. Towards a Directive on consumer rights. Munich: Sellier, 2010: 7-12.

Gsell, B.; Schellhase, H. M. Vollharmonisiertes Verbraucherkreditrecht - ein Vorbild für die weitere europäische Angleichung des Verbrauchervertragsrechts? JZ, 2009: 20-29.

Howells, G.; Reich, N. The current limits of European harmonisation in consumer contract law.ERA Forum, 2011/12: 39-57.

Howells, G.; Schulze, R. Overview of the Proposed Consumer Rights Directive. In
Howells, G.; Schulze, R. (Ed.) Modernising and Harmonising Consumer Contact Law. Munich: Sellier, 2009: 3-25.

Loos, M. Full harmonisation as a regulatory concept and its consequences for the national legal orders. The example of the Consumer rights directive. In Stürner, M. (Ed.) Vollharmonisierung im Europäischen Verbraucherrecht? München: Sellier, 2010: 47-98.

Loos, M. Rights of Withdrawal. In Howells, G.; Schulze, R. (Ed.) Modernising and Harmonising Consumer Contract Law. Munich: Sellier, 2009: 237-277.

Micklitz, H.-W. The targeted full harmonisation approach: looking behind the curtain. In Howells, G.; Schulze, R. Modernising and Harmonising Consumer Contract Law. Munich: Sellier, 2009: 47-86.

Micklitz, H.W.; Reich, N. Crónica de una muerte anunciada: The Commission Proposal for a 'Directive on Consumer Rights'. MLRev, 2009: 471-519.

Nordhausen Scholes, A. Information Requirements. In Howells, G.; Schulze, R. (Ed.) Modernising and Harmonising Consumer Contract Law. Munich: Sellier, 2009: 213-236. 
Paek, K.-I. Der Bürgenschutz im Spannungsfeld zur Privatautonomie. Hamburg: Verlag Dr. Kovač, 2006.

Reich, N. Economic Law, Consumer Interests and EU Integration. In: Micklitz, H.-W.; Reich, N.; Rott, P. Understanding EU Consumer Law. Antwerp: Intersentia, 2009: 1-60.

Rott, P.; Terryn, E. The proposal for a Directive on consumer rights: no single set of rules. ZEuP, 2009: 456-488.

Smits, J. M. Full Harmonization of Consumer Law? A Critique on the Draft Directive on Consumer Rights. European Review of Private Law, 2010, Vol. 18: 5-14.

Tonner, K.; Fangerow, K. Directive 2011/83/ EU on consumer rights: a new approach in European consumer law?EUVR, 2012/2: 6780.

Tonner, K.; Tamm, M. Der Vorschlag für eine Richtlinie über Verbraucherrechte und seine Auswirkungen auf das nationale Verbraucherrecht. JZ, 2009: 277-290.

Twigg-Flesner, C. "Time to do the job properly" - The case for a new approach to EU consumer legislation.Journal of Consumer Policy, 2010/33: 355-375.

Twigg-Flesner, C.; Metcalfe, D. The Proposed Consumer Rights Directive - less haste, more thought? European Review of Contract Law, 2009: 368-391.

Universität Bielefeld (Hrsg.), EC Consumer Law Compendium [interaktiv]. 2012 [aufgerufen: 2013-07-31]. <http://www.eu-consumer-law.org/index de.cfm>.

Universität Bielefeld (Hrsg.), EG-Verbraucherrechtskompendium [interaktiv]. 2007 [aufgerufen: 2013-07-30]. <http://www. eu-consumer-law.org/consumerstudy_full_ de.pdf $>$

Wendlandt, B. EC Directives for SelfEmployed Commercial Agents and on Time-Sharing - Apples, Oranges and the Core of the Information Overload Problem. In Howells, G.; Janssen, A.; Schulze, R. (Ed.) Information Rights and Obligations. Aldershot: Ashgate Publishing, 2005: 67-92.

Wilhelmsson, T. Full harmonisation of consumer law? ZEuP, 2008: 225-229.

\title{
NAUJIEJI ES VARTOTOJŲ TEISIŲ APSAUGOS STANDARTAI? NAUJA VARTOTOJŲ TEISIŲ DIREKTYVA 2011/83/ES
}

\author{
Arndt Künnecke
}

\author{
Okano universitetas, Stambulas, Turkija
}

Santrauka. Pastaraisiais metais vartotoju teise vis labiau ir labiau siejasi su ES įstatymu leidyba. Vienas iš svarbiausiu ES tikslu, baigiant sukurti vidaus rinka, yra vartotoju teisiu pozicionavimas šiame kontekste. Atsižvelgiant į įvairias pastaraisiais dešimtmečiais istatymais numatytas vartotoju apsaugos priemones, darytina išvada, kad ES èmesi nuodugniai peržiūreti savo vartotojų acquis. Po metu konsultaciju Vartotoju teisiu direktyva 2011/83/ ES, turejusi nustatyti naujus vartotoju apsaugos standartus, isigaliojo ir turés büti igyvendinta valstybese narese iki 2013 m. gruodžio $13 d$.

Atsisakydamas savo minimalaus suderinimo vartotoju teises srityje praktikos, t. y. leidžiant valstybems narems dèl direktyvose nurodytu pagrindu priimti griežtesnes apsaugos taisykles, ES teises aktu leidejas pasuko link visiško Vartotoju teisiu direktyvoje nustatytu priemoniu suderinimo, siekiant didinant vartotoju apsauga visoje ES, sutelkiant viename 
dokumente siuo metu skirtinguose istatymuose įtvirtintas nuostatas del nuotolines prekybos ir sutarčiu, sudarytu ne prekybai skirtose patalpose, taip pat kitu sutarčiu su vartotojais.

Straipsnyje glaustai supažindinama su šios direktyvos priemimo prielaidomis, aptariamos pagrindines harmonizavimo priemones. Taip pat analizuojama direktyvos apréptis, koncepcijos ir turinys, kadangi šis naujas teisés aktas inspiruoja dideles reformas reikalavimu. teikti informaciją ir teisés atsisakyti sutarties srityse. Atsižvelgiant į nustatytus direktyvos patobulinimus ir identifikuotus trükumus, daroma išvada, jog tolesniam harmonizavimui ir universalizavimui yra bütinas visuotinai taikomas Europos vartotoju teisiu rinkinys.

Reikšminiai žodžiai: vartotojų teisių apsauga, vartojimo sutarčiu teise, ES direktyvos, ES vartotoju teise, visiskas harmonizavimas.

\title{
NEW EU STANDARDS OF CONSUMER PROTECTION? NEW DIRECTIVE ON CONSUMER RIGHTS 2011/83/EU
}

\author{
Arndt Künnecke
}

Okan University, Istanbul, Turkey

Summary. In recent years consumer law has come more and more into the focus of legislation within the EU. One of the EU's key objectives, completing the final stage of the internal market, is to place consumer rights in the centre of it. Following the adaption of various consumer law measures for some decades, the EU has undertaken a thorough review of its consumer acquis. After years of consultations, the Consumer Rights Directive 2011/83/ $E U$, which was supposed to set new standards of consumer protection, came into force and will have to be implemented by the Member States by 13 December, 2013.

Renouncing its principal practice of minimum harmonisation in the area of consumer law, i.e. allowing Member States on the basis of Directives to adopt more protective rules, the EU legislator now turned to a targeted full harmonisation approach by means of the Consumer Rights Directive, aiming at increasing the consumer protection across the EU by bringing together the currently distinct laws for distance selling and off-premises contracts as well as other types of consumer contracts in a single instrument.

The article briefly introduces the background of the Directive and discusses the shift in means of harmonisation concepts. Then, it analyses the scope, concepts and content of the Directive, which mainly brings considerable reforms in the areas of information requirements and the right of withdrawal. Taking into account the Directive's improvements and shortcomings, conclusions are drawn that further harmonisation and a uniform and universally applicable set of European consumer rights are needed.

Keywords: Consumer Rights Protection, Consumer Contract Law, EU-Directives, EU Consumer Law, Full Harmonisation Approach. 
Arndt Künnecke, Okano universiteto (Stambulas, Turkija) docentas, teisės daktaras. Mokslinių tyrimų kryptys: mažumų teisès, lyginamoji teisè.

Arndt Künnecke, Okan University (Istanbul, Turkey), Assist. Prof. Dr., Lecturer. Research interests: minority rights, comparative law. 